\title{
Labyrinthe
}

18 | 2004 (2)

La Recherche dans tous ses éclats

\section{Antoine Prost, Jay Winter, Penser la Grande Guerre. Un essai d'historiographie}

Élise Julien

\section{OpenEdition}

1 Journals

Édition électronique

URL : http://journals.openedition.org/labyrinthe/215

DOI : 10.4000/labyrinthe.215

ISSN : 1950-6031

Éditeur

Hermann

Édition imprimée

Date de publication : 15 juillet 2004

Pagination : 53-68

Référence électronique

Élise Julien, «Antoine Prost, Jay Winter, Penser la Grande Guerre. Un essai d'historiographie », Labyrinthe [En ligne], 18 | 2004 (2), mis en ligne le 20 juin 2008, consulté le 01 mai 2019. URL : http://

journals.openedition.org/labyrinthe/215; DOI : 10.4000/labyrinthe.215

Propriété intellectuelle 


\title{
Penser la Grande Guerre. Un essai d'historiographie d'Antoine PROST et Jay WINTER*
}

\author{
Élise JuLIEN \\ elise-julien@infonie.fr
}

Antoine Prost et Jay Winter, les auteurs de Penser la Grande Guerre. Un essai d'historiographie, partent, dans leur introduction, du constat simple qu'un flot immense d'ouvrages a été consacré à la Première Guerre mondiale: cinquante mille sont ainsi inventoriés à la seule BDIC de Nanterre. Articles, films, émissions télévisées, expositions et sites Internet participent en outre à l'élaboration de l'histoire du conflit. Tous les éléments de cette production ont tenté de saisir une partie ou un aspect de la guerre, et cette histoire est bien loin d'être achevée. Cependant, personne ne s'est soucié de fournir une analyse d'ensemble de la façon dont s'est structurée l'histoire de la guerre, de mettre en évidence l'évolution des approches que celle-ci a développée et d'en déterminer les logiques sous-jacentes.

En effet, si des tentatives importantes ont été menées précédemment et que, à ce titre, Penser la Grande Guerre n'est pas le premier ouvrage à s'intéresser à l'historiographie de la Première Guerre mondiale, ces tentatives n'avaient à l'examen pas l'envergure de ce dernier ouvrage. On peut ici relever quelques exemples. L'essai de Jacques Droz sur les causes de la Première Guerre mondiale ${ }^{1}$ avait ainsi dressé dès 1973 un premier bilan de la bibliographie portant sur son sujet. Par ailleurs, dans le contexte de l'engouement mémoriel actuel, les commémorations ont souvent l'avantage d'inviter les historiens à la réflexivité. Pour le quatre-vingtième anniversaire de l'Armistice de 1918, un colloque se tenait à Montpellier sous le titre «La Grande Guerre 1914-1918. 80 ans

* Paris, Seuil, collection «Points histoire», série «L'Histoire en débats », 2004.

1. Les Causes de la Première Guerre mondiale, essai d'historiographie, Paris, Seuil, 1973. 
d'historiographie et de représentations ${ }^{2} \gg$. C'était l'occasion de constater l'accélération du rythme des parutions d'ouvrages consacrés à la guerre. Les organisateurs avaient surtout l'ambition d'offrir un panorama des nouvelles tendances de l'histoire militaire comparée, désormais ouverte à l'Europe, et d'aboutir à «la problématique d'une Grande Guerre débarrassée d'une analyse militaire dénuée de réflexion historique ». Avec ce programme, la rencontre ne pouvait en réalité permettre de saisir une historiographie qui s'est développée sur des fronts divers et pas seulement militaires, et qui s'est depuis longtemps souciée d'avoir des bases scientifiques. À défaut d'être novateur ${ }^{3}$, ce colloque présentait l'avantage de réunir en 1998 les tenants d'options historiographiques diverses, dont certaines paraissent aujourd'hui parfaitement antithétiques, mais ne les insérait pas dans une évolution et une lecture d'ensemble. À l'occasion d'un autre anniversaire, celui des dix ans d'existence de l'Historial de la Grande Guerre à Péronne (Somme), un colloque avait lieu en 2002 sous le titre: «Dix ans d'histoire culturelle de la Grande Guerre ${ }^{4} »$. Les contributions, variées et riches, concernaient pour partie les évolutions de l'historiographie et de ses cadres conceptuels et les divers modes de représentation de la guerre. Néanmoins, il s'agissait d'éclairer surtout les perspectives de l'histoire culturelle de la guerre. Finalement, ces trois tentatives, aux degrés d'intérêt divers, procèdent en quelque sorte du bilan sectoriel. Toutefois, par d'autres moyens, les initiatives en matière d'historiographie de la Grande Guerre se poursuivent. Après la vaste encyclopédie de la Première Guerre mondiale parue en Allemagne cet automne ${ }^{5}$, un dictionnaire critique est en préparation en France sous la direction de Stéphane Audoin-Rouzeau et de Jean-Jacques Becker. De telles entreprises traduisent plus une volonté de faire le point sur un champ de recherches qu'un désir d'analyser les évolutions et structures d'une historiographie ou d'en discerner les logiques.

2. Jules Maurin, Jean-Charles Jauffret (dir.), La Grande Guerre 1914-1918. 80 ans d'historiographie et de représentations, Montpellier, université de Montpellier III, 2002. Publication du colloque international du 20 au 21 novembre 1998.

3. Selon les espoirs de ses organisateurs : La Grande Guerre, op. cit., Présentation, p. 14.

4. Progamme disponible sur le site de l'Historial: http://www.historial.org/fr/centre/colloq2002a.htm

5. Gerhard Hirschfeld, Gerd Krumeich, Irina Renz, Enzyklopädie Erster Weltkrieg, Padeborn, Schöning, 2003, 1002 p. 


\section{Penser la Grande Guerre. Un essai d'historiographie}

Aucune de ces initiatives, ouvrage, colloque ou dictionnaire, n'a véritablement prétendu englober quatre-vingts ans d'historiographie ni en livrer une lecture articulée. Peut-être l'entreprise paraissait-elle irréalisable. C'est pourtant celle qu' ambitionnent de réaliser les auteurs de Penser la Grande Guerre, au-delà du souci de dresser également un état des lieux, et c'est ce qui fait le caractère novateur et l'enjeu intellectuel de ce livre.

Antoine Prost et Jay Winter sont deux éminents professeurs d'histoire, spécialistes de la Première Guerre mondiale, à qui l'on doit des œuvres de référence ${ }^{6}$. Ils sont partie prenante de l'écriture de cette histoire et mêlés d'une manière ou d'une autre aux débats récents qui l'animent'. Le souci permanent qu'ils ont d'offrir un cadre d'analyse général et de mettre leur objet à distance évite néanmoins à la présentation historiographique d'apparaître comme partisane. S'ils laissent parfois transparaître leurs propres choix, ils s'efforcent en tout cas de dépassionner les discussions. La collaboration de ce Français et de cet Américain facilite en outre la comparaison des différentes historiographies nationales et contribue, par cette approche croisée, à une meilleure appréciation des débats particuliers.

Les auteurs retiennent une acception large de l'histoire de la guerre: leur approche s'enrichit des apports d'autres disciplines, de la littérature à la sociologie; elle prend en compte l'ensemble du champ historiographique et non une histoire seulement politique, diplomatique ou militaire; elle analyse les historiographies française et anglo-saxonne, dans une moindre mesure allemande, et plus ponctuellement italienne. À partir de cet abondant matériau, il ne s'agit pas de produire un recensement ou une nomenclature mais bien de démêler et de décomposer différents «narratifs » afin de mieux mettre en place ce qui constitue les pièces

\footnotetext{
6. Antoine Prost est professeur émérite à l'université Paris I. Jay Winter a longtemps été professeur à Cambridge avant d'enseigner dorénavant à Yale. Antoine Prost a écrit sa thèse d'Etat sur les Anciens combattants: Les Anciens Combattants et la société française, Paris, Presses de la FNSP, 1977, 3 volumes. Jay Winter s'est intéressé à de nombreux aspects de la société britannique dans la guerre, ainsi qu'à la dimension mémorielle de celle-ci. Voir notamment: The Experience of World War I, London, Macmillan, 1988 ; traduction française: La Grande Guerre, éclatement d'un monde, Paris, Readers Digest, 1990, et Sites of Memory, Sites of Mourning. The Great War in European Cultural History, Cambridge, Cambridge University Press, 1995.

7. Jay Winter est membre du comité directeur du Centre de recherche de l'Historial de la Grande Guerre à Péronne; Antoine Prost intervient régulièrement dans les revues scientifiques sur des sujets relevant de l'histoire et de l'historiographie de la Première Guerre mondiale.
} 
d'un puzzle historiographique et de renouer les fils d'histoires et d'objets multiples. Il en ressort des analyses remarquables et une vue d'ensemble articulée autour de trois configurations historiques générales.

La première de ces configurations, «militaire et diplomatique», est élaborée dès la guerre par des acteurs, dirigeants politiques et militaires, autant que par des essayistes et des historiens professionnels. Elle est dominée par des enjeux historiographiques directement liés à la politique, et les recherches se concentrent alors notamment autour de la question centrale des responsabilités. Dans cette configuration, l'histoire de la guerre est nationale et elle compte sur la méthode critique pour accéder à la légitimité; elle s'attache de ce fait aux événements les mieux vérifiables, aboutissant à faire de la guerre un objet vu d'en haut, qui laisse peu de place aux sociétés et aux combattants.

Une deuxième configuration, «sociale», se fait jour après la Seconde Guerre mondiale. Sous l'influence diffuse du marxisme et dans le contexte d'un marché de l'histoire en expansion, le regard historien sur la guerre se fait plus global. Ses intérêts se déplacent, des étatsmajors à la vie des soldats et des diplomates à la vie des civils, et parmi eux des ouvriers. Une guerre se dessine alors où l'économique et le social trouvent une large place.

À partir de cette approche on aboutit, par glissement progressif des centres d'intérêt, à une troisième configuration qualifiée de «culturelle et sociale». La transition est facile, car l'histoire culturelle était depuis longtemps présente dans l'histoire sociale, et même dans l'histoire politique; elle est désormais revendiquée pour servir de paradigme structurant. L'histoire de la guerre se fait ainsi par en bas; elle se penche sur des cas particuliers pour saisir des expériences et se diversifier. Elle interroge aussi les objets, le vécu intime de la guerre, les pratiques liées à la violence ou au deuil. Ce «front pionnier» que demeure l'histoire culturelle du conflit est aussi celui qui occupe l'actualité.

Par cette évolution d'ensemble, exposée dans les deux chapitres qui ouvrent et ferment le livre, l'historiographie de la Grande Guerre fournit un point de vue sur l'évolution plus générale de l'écriture de l'histoire au $\mathrm{XX}^{\mathrm{e}}$ siècle. Mais cette historiographie peut aussi, à l'inverse, être déclinée pour chacune des questions se rapportant à la Première Guerre mondiale. C'est pourquoi les sept chapitres centraux sont thématiques et traitent de l'historiographie de chaque secteur de l'histoire de la guerre. Les auteurs y posent les grandes questions auxquelles cette 


\section{Penser la Grande Guerre. Un essai d'historiographie}

histoire a été confrontée: pourquoi et pour quoi la guerre? Qui commande et comment? Qu'est-ce que faire la guerre ? Comment faire une guerre industrielle? Quels liens entre guerre et révolution? Pourquoi les civils ont-ils tenu? Comment vivre quand on ne peut ou ne veut oublier? Ces interrogations contenues dans les titres de chaque chapitre permettent respectivement d'étudier les cabinets et ambassades, les quartiers généraux et ministères, les tranchées, les économies, les usines, l'arrière, les discours sur la guerre. Les auteurs présentent alors pour chaque cas les nuances et l'articulation spécifique des différentes configurations évoquées plus haut; ils exposent en outre les résultats, les lacunes et les débats propres à chaque champ.

Ainsi, pour ne prendre qu'un exemple, la recherche sur l'arrière et les civils s'est-elle développée selon trois axes successifs: les masses en guerre, les structures sociales, les cultures. L'intérêt de l'histoire pour la vie civile est réel dès le lendemain de la guerre, mais il s'agit d'une histoire par les officiels, où les populations sont des masses passives. Les études se concentrent sur la gestion des villes en guerre; elles sont en revanche quasi-inexistantes sur les populations rurales; il n'est en outre pas encore question de comparaisons transnationales. En écho à des événements contemporains, de manière variable selon les pays, les années 1960 et 1970 voient s'approfondir l'étude du rapport entre guerre et société. On en apprend alors davantage sur les conditions matérielles de vie et sur la démographie du temps de guerre par des études qui s'inspirent de la sociologie weberienne et marxiste, et qui se situent dans la continuité d'une histoire sociale quantitative. Enfin, la dernière génération de travaux sur l'arrière tend vers une histoire anthropologique qui s'attache à comprendre les racines du consensus affectant l'arrière, notamment par la notion de culture de guerre.

Au-delà des conclusions spécifiques à chaque champ de recherche, il se dégage de l'ouvrage trois manières principales de penser la guerre: ce sont tour à tour les nations, les sociétés, les gens qui se sont trouvés au cœur de l'analyse. Ce sont aussi trois générations d'historiens qui se sont succédé. Dans ce mouvement général, les approches nationales conservent une autonomie et des spécificités qui tiennent aux cadres et aux pré-requis de la recherche: victoire douloureuse en France, la guerre est une défaite longtemps niée en Allemagne et une absurdité en Grande-Bretagne. 
Ce livre, par la densité de ses analyses et le nombre de sujets qu'il aborde, grâce aussi au parti pris d'une approche croisée internationale, offre un impressionnant panorama des travaux et des débats qui ont nourri et accompagné l'écriture de l'histoire de la guerre. La méthode d'exposition - présentation des configurations générales puis déclinaison pour chaque champ de recherche - amène cependant à des redondances. Cette structure «en mille-feuilles» rend la lecture linéaire peu confortable, mais peut-être est-ce le prix à payer pour obtenir une vue d'ensemble satisfaisante, qui rende compte à la fois des tendances historiographiques lourdes, des variations plus ou moins marquées que connaissent les différents champs de la recherche, et du parcours sinueux pour mettre ces deux pôles en relation constante. En revanche, on dispose d'un excellent outil de travail, que l'on peut consulter au gré de ses intérêts sans crainte d'en épuiser trop vite les ressources.

Comment, en conclusion, «penser la Grande Guerre»? Il n'est évidemment pas question pour Antoine Prost et Jay Winter de tenter de fournir une réponse normative à la question. Leur dessein historiographique les engageait seulement - mais au prix de quel travail - à mettre au jour une cohérence dans le désordre de l'écriture historique. Ils n'ont pas épuisé le sujet, ne serait-ce que parce que l'histoire de la guerre n'est pas achevée. Mais la voie qu'ils ont ouverte est indispensable au progrès de la réflexion sur la façon dont l'historiographie ne cesse de se redéployer et dont l'histoire participe à la gestion collective du passé. Les deux auteurs parviennent à démêler les «narratifs» des diverses histoires de la guerre, pour finalement conclure à l'impossibilité de la synthèse par une histoire totale: les façons de penser la guerre sont diversifiées et chacune légitime, sans qu'aucune n'ait lieu de dominer vraiment. Ce constat, relativiste, d'une pluralité irréductible des histoires pourrait paraitre pessimiste. Pour les auteurs du livre, elle invite surtout à l'humilité, par eux pleinement acceptée, de l'historien face à cet événement. Et l'on retrouve pour finir la conviction, désormais solidement fondée, par laquelle ils ouvraient leur livre: «La guerre de 1914 n'appartient à personne, pas même aux historiens.» 